\title{
Heterogeneous Responses of Tonically Active Interneurons in the Dorsal Striatum
}

\author{
Eric Garr \\ Program in Animal Behavior and Comparative Psychology, Graduate Center, City University of New York, New York, New York 10016 \\ Review of Nougaret and Ravel
}

The basal ganglia are an important set of subcortical nuclei that control behavioral output during instrumental learning and decision-making. The largest part of the basal ganglia, the striatum, is made up mostly of medium spiny neurons (MSNs) that indirectly modulate motor cortical areas. The striatum also contains a set of tonically active interneurons (TANs) presumably cholinergic - that modulate MSN excitability and receptivity to cortical input (for review, see Schulz and Reynolds, 2013). At the population level, these interneurons respond to motivationally relevant stimuli with a pause in firing followed by a subsequent increase in firing above baseline, although there is evidence to suggest that perhaps the patterns observed in the population do not accurately represent the responses of individual cells (Joshua et al., 2008). It is unclear exactly how these neurons contribute to reward-guided behavior, but it is clear that disrupted striatal cholinergic function characterizes various movement disorders (Deffains and Bergman, 2015). It is thus essential for researchers to study how TANs fire under conditions where movement is required to obtain reward.

Received Jan. 10, 2016; revised Feb. 17, 2016; accepted Feb. 20, 2016.

I thank Jon C. Horvitz for helpful comments.

The author declares no competing financial interests.

Correspondence should be addressed to Eric Garr, Department of Psychology, Brooklyn College, City University of New York, William James Hall, Room 4401B, Brooklyn, NY 11210. E-mail: egarr@gradcenter.cuny.edu.

DOI:10.1523/JNEUROSCI.0099-16.2016

Copyright $\odot 2016$ the authors $\quad 0270-6474 / 16 / 363412-02 \$ 15.00 / 0$
In a recent study published in The Journal of Neuroscience, Nougaret and Ravel (2015) examined how TANs responded in two monkeys trained to press a lever for water reward in the presence of the following two cues: one that signaled the amount of force required for a successful operant response (high or low force); and one that signaled the magnitude of the reward to be earned (high or low reward). Thus, on each trial, one of the following four cue combinations were presented: low force/high reward; high force/high reward; low force/low reward; or high force/low reward. After training, data were collected from extracellular recordings of TANs, the majority of which were in the caudate nucleus.

The main analysis was concerned with how neuronal activity was modulated by trial type during the cue presentation compared with reward delivery. Many TANs showed a pause or increase in firing in response to the cue and/or the reward. Interestingly, separate groups of TANs were modulated during the two task epochs-TANs that paused in response to cue presentation were generally not the same cells that paused in response to reward delivery, and the same was true for increases in firing. While few TANs showed a pause during cue presentation, many showed an elevation response. In contrast, following the delivery of reward, a similar number of TANs responded with pauses and elevations. Notably, neuronal activity following cue presentation was generally better aligned to the operant re- sponse, with the majority of pauses preceding the lever press and the majority of elevations coinciding with the lever press.

The magnitude of the pauses and elevations during the cue and reward epochs were dependent on the trial type, with separate groups of TANs modulated by the amount of required force and the amount of reward. Of the TANs showing force-modulated elevations during cue presentation, the vast majority were modulated during the high-force trials. These neurons were separate from those showing reward magnitude-modulated elevations during cue presentation, the vast majority of which were modulated during low-reward trials. Thus, immediately following cue presentation, two separate groups of TANs showed an increased firing rate- one during high-force trials and one during low-reward trials. As for the onset of reward, most force-modulated pauses were modulated during the lowforce trials, while reward magnitudemodulated pauses were distributed evenly between high- and low-reward trials. An approximately even number of TANs showing force-modulated elevations at the time of reward delivery were split between high- and low-force trials. These neurons were distinct from those showing reward magnitude-modulated elevations, the majority of which were modulated during low-reward trials.

The upshot of these findings is that different populations of TANs respond during particular task epochs and only during particular trial types. While it is tempting 
to conclude that the pauses and elevations encode motivational variables such as effort and subjective value, it is also possible that the modulations reflect information about the identity of the trial type independent of the motivational value. If this is true, TANs might be responsible for encoding the identity of various trial types and using these "state representations" to compute identity prediction errors when stimuli or contingencies differ from expectation (Bradfield et al., 2013; Schoenbaum et al., 2013). In the context of the present study, these proposed state representations would most likely be achieved via the TAN elevation response, because the magnitude of elevation responses, rather than of pauses, was most influenced by trial type. However, further work is needed to determine whether the elevation responses actually encode identity or whether they encode motivational value, as the authors suggest. While learning theories have mostly focused on the baseline tonic activity and the stereotyped pause response of TANs (Ashby and Crossley, 2011), the elevation response is clearly modulated by motivationally relevant stimuli and is relevant to instrumental performance.

The potential downstream consequences of increases in TAN firing rates are complex. Synchronized firing among TANs triggers presynaptic release of acetylcholine onto nicotinic receptors of dopaminergic terminals in the striatum, which results in dopamine release (Threlfell et al., 2012). Dopamine is critical for potentiating and weakening corticostriatal synapses at MSNs expressing $D_{1}$ and $D_{2}$ receptors, respectively. These alterations in plasticity only occur, however, when MSNs are also highly depolarized (Surmeier et al., 2009). It is therefore interesting that Nougaret and Ravel (2015) found that TAN elevation responses coincided with lever presses, because MSNs have also been shown to fire phasically in response to instrumental actions (Hollerman et al., 1998). It is possible that coincidental firing in striatal TANs and MSNs, combined with stimulus-evoked cortical firing, could serve to reinforce the pressing actions required to obtain reward in the task.

On the other hand, there are multiple mechanisms by which acetylcholine re- lease from TANs can inhibit MSNs (English et al., 2011; Nelson et al., 2014). This inhibitory cholinergic influence within the striatum has been hypothesized to promote action selection by inhibiting competing responses (Stocco, 2012). It is possible that the TAN elevation response served such a function at the time of the lever press in the current study, especially considering the fact that monkeys were required to hold the lever down for $1 \mathrm{~s}$ to receive reward and thus inhibit other irrelevant actions. The response inhibition hypothesis could also help to explain why the motivationally unattractive trials (i.e., high force and low reward) were more likely to evoke elevation responses across separate TAN populations. The high-elevation responses observed during these trials may have been necessary to inhibit competing actions such as applying too much force to the lever or releasing the lever too early-erroneous responses that were relatively common in these types of trials.

The findings presented by Nougaret and Ravel (2015) call for a revision to the notion that TAN activity is uniform across the neural population. There appears to be a remarkable amount of heterogeneity, suggesting that it is unwise to draw conclusions from averaged population activity. Not only do separate groups of TANs respond to the cue and reward, but there also appear to be neurons within each population that respond in an idiosyncratic way, depending on the trial type. Furthermore, the fact that the pauses and elevations were better aligned to the operant response rather than the onset of the cue indicates that TANs ought to be more carefully studied in relation to action execution. Finally, although the results of the present study show that the TAN elevation response is modulated by trial type, the function of this response in the context of behavior remains a mystery. Further research will be required to elucidate exactly how TAN pauses and elevations affect MSNs and striatal afferents, and how these interactions influence reward-guided behavior.

\section{References}

Ashby FG, Crossley MJ (2011) A computational model of how cholinergic interneu- rons protect striatal-dependent learning. J Cogn Neurosci 23:1549-1566. CrossRef Medline

Bradfield LA, Bertran-Gonzalez J, Chieng B, Balleine BW (2013) The thalamostriatal pathway and cholinergic control of goal-directed action: interlacing new with existing learning in the striatum. Neuron 79:153-166. CrossRef Medline

Deffains M, Bergman H (2015) Striatal cholinergic interneurons and cortico-striatal synaptic plasticity in health and disease. Mov Disord 30:1014-1025. CrossRef Medline

English DF, Ibanez-Sandoval O, Stark E, Tecuapetla F, Buzsáki G, Deisseroth K, Tepper JM, Koos T (2011) GABAergic circuits mediate the reinforcement-related signals of striatal cholinergic interneurons. Nat Neurosci 15: 123-130. CrossRef Medline

Hollerman JR, Tremblay L, Schultz W (1998) Influence of reward expectation on behaviorrelated neuronal activity in primate striatum. J Neurophysiol 80:947-963. Medline

Joshua M, Adler A, Mitelman R, Vaadia E, Bergman $\mathrm{H}$ (2008) Midbrain dopaminergic neurons and striatal cholinergic interneurons encode the difference between reward and aversive events at different epochs of probabilistic classical conditioning trials. J Neurosci 28:11673-11684. CrossRef Medline

Nelson AB, Hammack N, Yang CF, Shah NM, Seal RP, Kreitzer AC (2014) Striatal cholinergic interneurons drive GABA release from dopamine terminals. Neuron 82:63-70. CrossRef Medline

Nougaret S, Ravel S (2015) Modulation of tonically active neurons of the monkey striatum by events carrying different force and reward information. J Neurosci 35:15214-15226. CrossRef Medline

Schoenbaum G, Stalnaker TA, Niv Y (2013) How did the chicken cross the road? With her striatal cholinergic interneurons, of course. Neuron 79:3-6. CrossRef Medline

Schulz JM, Reynolds JN (2013) Pause and rebound: sensory control of cholinergic signaling in the striatum. Trends Neurosci 36: 41-50. CrossRef Medline

Stocco A (2012) Acetylcholine-based entropy in response selection: a model of how striatal interneurons modulate exploration, exploitation, and response variability in decision-making. Front Neurosci 6:18. CrossRef Medline

Surmeier DJ, Plotkin J, Shen W (2009) Dopamine and synaptic plasticity in dorsal striatal circuits controlling action selection. Curr Opin Neurobiol 19:621-628. CrossRef Medline

Threlfell S, Lalic T, Platt NJ, Jennings KA, Deisseroth K, Cragg SJ (2012) Striatal dopamine release is triggered by synchronized activity in cholinergic interneurons. Neuron 75:58-64. CrossRef Medline 